\title{
Schizophrenic hypothesis behavior in concept identification
}

\author{
VLADIMIR PISHKIN \\ Veterans Administration Hospital and University of Oklahoma Health Sciences Center, \\ Oklahoma City, Oklahoma 79104 \\ and \\ W. VAIL WILLIAMS \\ Mental Research Institute, Palo Alto, California 94304
}

\begin{abstract}
A concept identification (CI) task designed for analysis of hypothesis behavior was administered to a total of $\mathbf{1 0 0}$ chronic undifferentiated schizophrenic and nonpsychiatric control patients matched on age, IQ, and length of hospitalization. Major results were: (a) Controls were more efficient in utilizing relevant information as stimulus complexity increased; (b) focusing was adopted by controls as compared to random scanning strategy by schizophrenics; (c) analysis of hypothesis behavior was based on 10 strategy characteristics utilized in CI which demonstrated schizophrenic deficit in cognitive strategy.
\end{abstract}

Cognitive deficit in schizoprenic subjects has been documented across a broad spectrum of theoretical orientations utilizing a number of tests of thinking ability. In order to explore the nature of the deficit within the information processing sphere, Cameron's (1947) ideas regarding overinclusion were instrumental in generating research within the concept identification (CI) paradigm, whereby the amounts of relevant and irrelevant information loads were quantitatively manipulated (Pishkin \& Blanchard, 1963). On the whole, inability of schizophrenics to screen out irrelevant information in CI has been reliably demonstrated (Pishkin \& Bourne, 1969). The present study was designed to evaluate the ability of schizophrenics and normals to selectively choose hypotheses or strategies of CI solution, in addition to concomitantly filtering relevant and irrelevant information. More importantly, there are virtually no data dealing directly with CI hypothesis behavior in patients diagnosed as schizophrenic, although there is some work related to this area with college students (Bourne, 1963). The importance of studying strategies has been emphasized by Van de Geer and Jaspers, who suggested that cognitive processes can be differentiated from simple learning processes in their emphasis on strategies: When "the individual is selectively collecting inputs in order to arrive at a final or semifinal decision, he brings with him a view of his own in dealing with the environment" (1966, p. 147).

Hypothesis behavior theories were first developed in the context of tasks which are simpler than conceptual problems. Krechevsky (1932) produced some convincing evidence on hypothesis-like behavior in rats on a simple

Authors are indebted to Louis M. Jones and Elizabeth A. Rasmussen for assisting with the data. This research was supported by Veterans Administration funds, MRIS 7089-07. two-choice discrimination task. Levine (1966) has adopted certain features of Krechevsky's analysis and coupled them with Harlow's (1959) error factor notions to produce a more explicit model of hypothesis behavior in humans. Hypothesis testing models of $\mathrm{CI}$ have been further extended by Trabasso and Bower (1968). Considerable work was done on strategies for concept learning by Bruner, Goodnow, and Austin (1956), who obtained four kinds of strategies: "conservative focusing," "focus gambling," "successive," and "simultaneous scanning." These strategies are referred to as selection strategies, e.g., the subject selects his own instances of what he thinks the concept is. Several investigators, rather than rely on the selection paradigm, have used the reception approach whereby the subject has no opportunity to select stimuli and must encode an experimentally regulated flow of information. Bruner et al. (1956), in using this framework, reported two effective strategies: the "wholist" and the "partist" strategies. Subsequently, Bourne (1963) reported results which were in accord with those of Bruner et al. and found that the "wholist" strategy was used in only 9\% of the problems. Furthermore, Bourne (1965), using the reception paradigm, found that the efficient learner starts with a more encompassing initial hypothesis (wholist), changes it only after he makes a category error, and changes the hypothesis in only one respect at a time. Also, the more efficient learners' hypotheses tend to be more consistent with previously given information. Since most CI tasks are based upon a reception paradigm, it would appear that Bourne's (1965) analysis of hypothesis behavior in relation to category responses could be applied to schizophrenics' performance on CI problems. In brief, Bourne's (1965) findings show that there are significant differences between more efficient and less efficient learners in terms of their hypothesis 


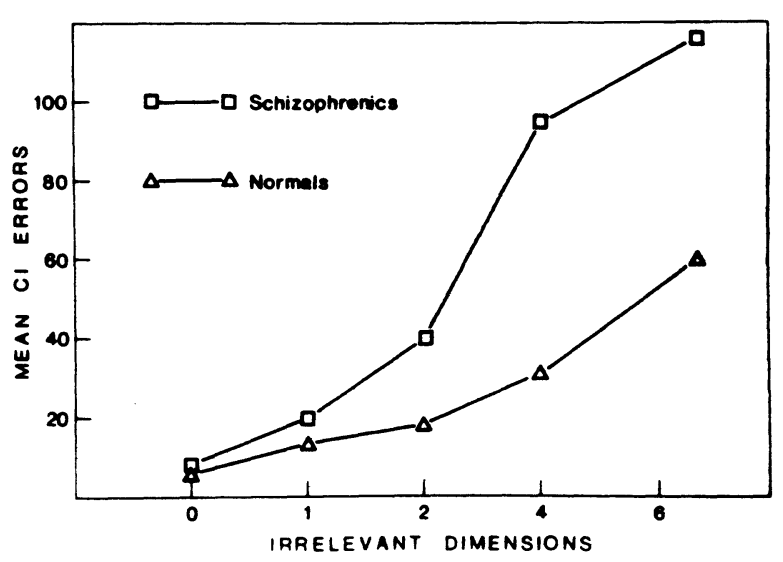

Figure 1. Mean concept identification errors by schizophrenic and normal subjects at five levels of complexity.

behavior. Accordingly, it was anticipated that normal subjects would start with a more encompassing initial hypothesis, keep the same hypothesis after a correct response more frequently, and be more consistent with previously presented information than would schizophrenic subjects. This assumption was based on the findings relating to schizophrenics' limited channel capacity and lack of selective focusing capability necessary for efficient information processing (Pishkin \& Bourne, 1969).

\section{METHOD}

\section{Subjects}

All subjects were inpatients at the Veterans Administration Hospital, Oklahoma City, Oklahoma. The schizophrenic sample consisted of 50 hospitalized white male patients (mean age 38.12 years), diagnosed as chronic undifferentiated schizophrenics, who were receiving phenothiazine medications not exceeding a daily dosage of $400 \mathrm{mg}$ of chloropromazine or its equivalent. The normal group (mean age 38.46 years) was composed of 50 hospitalized white male patients from orthopedic and general medical wards. Both groups were matched on the vocabulary and abstract Shipley Institute of Living scales and on age, intelligence, and length of hospitalization, to the extent that no significant difference occurred between any pair of groups for these variables. Both schizophrenic and normal samples excluded patients who suffered from organic brain syndrome, were drug or alcohol abusers, or had had electroconvulsion therapy.

\section{Procedure}

The procedural aspects of the two-choice CI tasks, apparatus, and instructions were the same as in an earlier study, described in detail by Pishkin (1960), with the exception that subjects indicated their hypotheses as to what the relevent dimensions were prior to making category responses. After a self-determined time, the subject indicated his hypothesis regarding which dimension(s) was relevant by pressing one or more stimulus dimension keys (number, color, horizontal/vertical position, form, size, and orientation were the possible relevant dimensions). This response was followed by depressing one of the two category response keys. Either form or color was the relevant dimension for half of the subjects in each condition, i.e., the subjects were to categorize all patterns according to either form or color and to ignore the irrelevant dimensions. Finally, it should be noted that the stimulus dimensions (labeled hypothesis keys) were always available to the subject. In this way,
Pishkin (1965) has shown that not only does performance on a CI task improve, but also the availability of the dimensions serves to limit the number of possible hypotheses the subject must consider. The depressed stimulus dimension keys stayed in a down position (continuous signal) until the subject pressed one of the two category response keys.

\section{Design}

This was a 5 by 2 factorial design: five levels of complexity (C); $0,1,2,4$, or 6 irrelevant dimensions and two groups (G); chronic undifferentiated schizophrenics or normals. The criterion for solution for all subjects was 16 consecutive correct responses or a maximum of 192 trials.

\section{RESULTS AND DISCUSSION}

Anaiysis of variance of errors to criterion showed that the main effects of $C(F=12.03, \mathrm{df}=4 / 90$, $\mathrm{p}<.001), \mathrm{G}(\mathrm{F}=7.86, \mathrm{df}=1 / 90, \mathrm{p}<.01)$, and the $\mathrm{C}$ by $\mathrm{G}$ interaction $(\mathrm{F}=3.67, \mathrm{df}=4 / 90, \mathrm{p}<.01)$ were all significant. As levels of complexity increased, performance became poorer for both groups. Schizophrenics had more errors than normals and showed a faster degradation of performance as complexity increased.

The analysis of hypothesis behavior in the present study was the same as that used by Bourne (1965). Ten basic characteristics of each subject's hypothesis behavior were determined: the number of dimensions in the subject's initial hypothesis (I), the number of times the subject changed his hypothesis in any way after making an incorrect category response (EC), the number of times after an error the subject did not change his hypothesis (EN), the number of times the subject changed his hypothesis after making a correct response (C), the number of times the subject did not change his hypothesis after making a correct decision $(\mathrm{N})$, the addition (A) or deletion (D) of dimensions from the subject's hypothesis of the previous trial (hypothesis shifts), increases in hypothesis size (A or $A>D$ ), decreases in hypothesis size ( $D$ or $D>A$ ), and changes in hypothesis composition only $(\mathrm{A}=\mathrm{D})$. The last five characteristics dealt with the subject's hypothesis shifts from a previous trial. Except for I, the frequency of each of the foregoing characteristics was converted to a proportion. This was done by dividing each characteristic by the total number of times that particular characteristic happened in the stimulus sequence shown to the subject. For example, EC and $\mathrm{C}$ were each divided by the total number of hypothesis changes displayed by the subject (in addition, EC and C were divided by the total number of incorrect and correct responses, respectively, resulting in $\mathrm{EC}_{1}$ and $\mathrm{C}_{1}$ ); $\mathrm{EN}$ and $\mathrm{N}$ were divided by the total number of errors and correct responses, respectively; and $\mathrm{A}, \mathrm{D}, \mathrm{A}>\mathrm{D}, \mathrm{D}>\mathrm{A}$, and $\mathrm{A}=\mathrm{D}$ were divided by the total number of changes after error. Finally, the proportion of hypothesis shifts of the various types that were consistent with previously presented information given to a subject in the series were computed. Then the mean value for each of these 
proportions was determined separately for normal and schizophrenic subjects in each experimental condition.

Analysis of hypothesis behavior was conducted on the five levels of complexity as well as with complexity conditions pooled for each group.

With complexity levels combined, normal subjects stayed with a particular hypothesis when they were correct $(\mathrm{N})$ more frequently than did schizophrenics $(\mathrm{t}=2.05, \mathrm{df}=98, \mathrm{p}<.05)$, and they were more consistent with previously presented information; following an error they added a dimension (A) to their hypothesis $(\mathrm{t}=2.37, \mathrm{df}=98, \mathrm{p}<.05)$. It is noteworthy that schizophrenics had higher percentages of shifts in their hypotheses after both an error $\left(\mathrm{EC}_{1}\right)$ and a correct response $\left(\mathrm{C}_{1}\right)$.

On the other hand, schizophrenics at the 2-irrelevant level had a higher percentage of changes after an incorrect response $\left(\mathrm{EC}_{1}\right)$ than did normals $(\mathrm{t}=2.23 \mathrm{df}=18$, $\mathrm{p}<.05$ ); at the 4-irrelevant level they kept their hypothesis after an error (EN) more frequently than did normals $(\mathrm{T}=2.26, \mathrm{df}=18, \mathrm{p}<.05)$; at the 6-irrelevant level they changed their hypothesis after a correct response $(\mathrm{C})$ more frequently than did normals $(\mathrm{t}=2.31$, $\mathrm{df}=18, \mathrm{p}<.05)$.

In terms of irrelevant dimension levels, normals tended to be more consistent with previously presented information at the 0 -irrelevant level when they added (A) a dimension to the hypothesis $(\mathrm{T}=2.89, \mathrm{df}=18$, $\mathrm{p}<.02$ ). Furthermore, at the 4-irrelevant level, normals were more consistent when, following an error, they changed the composition $(\mathrm{A}=\mathrm{D})$ of their hypothesis $(t=2.61, d f=18, p<.02)$. Finally, in terms of conditions involving the same amount of stimulus uncertainty, differences between normals' and schizophrenics' hypothesis behavior $\left(\mathrm{EC}_{1}\right)$ emerged mainly in those conditions involving 6 bits of irrelevant information. Here, schizophrenics had higher percentages of shifts after errors $(\mathrm{t}=2.71, \mathrm{df}=18, \mathrm{p}<.02)$ and after correct responses $(t=2.73$, df $=18, p<.02)$ than did normals. The higher percentage of shifts after errors also reached significance in those conditions involving two irrelevant dimensions $(t=2.92, \mathrm{df}=18, \mathrm{p}<.01)$. Furthermore, schizophrenics changed the composition of their hypothesis $(A=D)$ in the 6-irrelevant dimension more frequently than did normals $(\mathrm{t}=2.75, \mathrm{df}=18, \mathrm{p}<.05)$.

It is interesting to note that most of the significant differences in hypothesis behavior between schizophrenics and normals emerge in those conditions where maximum complexity is present in the stimulus series presented to each subject. In order to determine if each population group approached the task in a similar manner, percentages were computed based upon each subject's hypothesis responding in an attempt to isolate the relevant from the irrelevant information. It was shown that $76 \%$ of the normals' hypotheses centered on a relevant dimension for solution of the problem, while only $54 \%$ of the schizophrenics' hypotheses centered on a relevant dimension $(\mathrm{t}=2.61, \mathrm{df}=98, \mathrm{p}<.02)$. In the main, schizophrenics showed higher percentages of hypothesis shifts after both errors and correct responses than did normals. Furthermore, these shifts were primarily changes in composition of the hypotheses. In this respect, it appeared that schizophrenics were randomly choosing dimensions among the stimuli presented to them. If it is true that schizophrenics did not attend selectively to stimuli and tended to be more distractable, then, in the face of many irrelevant dimensions, they may have adopted a strategy of shifting back and forth in a vain attempt to organize the information. On the other hand, normals kept the same hypotheses more frequently when they were correct; when they added a dimension to their hypotheses, they were more consistent with previous information. In this respect, normals appeared to be "focusers," in that they adopted a strategy of retaining the hypothesis that worked better than chance and then introduced corrections designed to discover the remaining correct solution. This interpretation received some additional support in that normals' hypotheses centered on a relevant dimension to a greater degree than those of schizophrenics.

Based on the hypothesis behavior of normals and schizophrenics, both groups tended to adopt a partist approach, with the normals doing more focusing and the schizophrenics more scanning. This finding regarding the schizophrenic group lends support to Silverman (1963), who reported that extreme scanning characterizes the attention response types of most schizophrenics. Furthermore, in the present study, schizophrenics' hypothesis behavior can be characterized: They do not change their hypotheses after an error to the same degree as normals, have a higher percentage of change after a correct response as well as after errors, and are less consistent with previous information when they do make a change. On the other hand, the normals usually keep their hypotheses when correct, change them mainly when they make an error, generally change their hypotheses in one respect at a time, and their hypotheses tend to be more consistent with previously presented information.

Two distinct stages of efficient hypothesis behavior that may occur in a CI task are suggested. The first stage is that of changing the hypothesis frequently, though not necessarily on every error. This approach seems to be used until a hypothesis results in performance better than chance. The second stage consists of retaining the hypothesis which works most often and attempting to discover the complete hypothesis for correct solution, by making minor shifts or adjustments to the hypothesis. Once this solution is obtained, the subject makes no further hypothesis changes. Almost all of the normals appeared to efficiently follow such stages, while the schizophrenic subjects appeared to have difficulty with the second stage. Efficient performance would seem to depend upon proper use of previous information provid- 
ed in the task, that is, the ability to store past instances of the concept; it would seem to depend upon not changing a hypothesis after a correct response and changing the hypothesis only in error. Present data clearly demonstrate schizophrenics' deficit in ability to utilize relevant cues and to filter out distracting, irrelevant information. Schizophrenics simply do not profit from their past errors and have difficulty focusing on the reinforced cues. It is also noteworthy that the cognitive deficit in chronic undifferentiated schizophrenics may be viewed not only in terms of their inability to effectively integrate mnemonic and feedback information, but also in an ineffective set of strategies for making use of feedback.

\section{REFERENCES}

Bourne, L. E., JR. Some factors affecting strategies used in problems of concept formation. American Journal of Psychology, 1963, 76, 229-238.

BouRNE, L. E., JR. Hypotheses and hypothesis shifts in classification learning. Journal of General Psychology, 1965, 72, 251-261.

Bruner, J. S.. Goodnow. J. J., \& Austin, G. A. A study of thinking. New York: Wiley, 1956.

Cameron, N. The psychology of behavior disorders. Boston: Houghton Mifflin, 1947.
HARLOW, H. F. Learning set and error factor theory. In S. Koch (Ed.), Psychology: A study of a science (Vol. 1). New York: McGraw-Hill, 1959.

KRECheVSKY, I. "Hypotheses" in rats. Psychological Review, 1932, 38, 516-532.

LeViNe, M. Hypothesis behavior by humans during discrimination learning. Journal of Experimental Psychology, 1966, 71, 331-338.

PishKin, V. Effects of probability of misinformation and number of irrelevant dimensions upon concept identification. Journal of Experimental Psychology, 1960, 59, 371-378.

Pishoin, V. Dimension availability with antecedent success or failure in concept identification. Psychonomic Science, 1965 , 2, 69-70.

Pishkin, V. \& Blanchard, R. J. Stimulus and social cues in concept identification of schizophrenics and normals. Journal of Abnormal and Social Psychology, 1963, 67, 454-463.

Pishkin, V., \& Bourne, L. E., Jp. Concept identification by schizophrenic and normal subjects as a function of problem complexity and relevance of social cues. Journal of Abnormal Psychology, 1969, 74, 314-320.

Silverman, J. Psychological deficit reduction in schizophrenia through response-contingent noxious reinforcement. Psychological Reports, 1963, 13, 187-210.

Trabasso, T., \& Bower, G. H. Attention in learning. New York: Wiley, 1968.

Van De Geer, J. P., \& Jaspers, J. M. F. Cognitive functions. Annual Review of Psychology, 1966, 17, 145-176.

(Received for publication July 16, 1976.) 